\title{
On the Enhanced Temporal Coherency of Radar Observations in Precipitation
}

\author{
A. R. JAMESON \\ RJH Scientific, Inc., El Cajon, California \\ A. B. KosTINSKI \\ Michigan Technological University, Houghton, Michigan
}

(Manuscript received 28 September 2009, in final form 30 March 2010)

\begin{abstract}
In this work, the authors present observations of enhanced temporal coherency beyond that expected using the observations of the standard deviation of the Doppler velocities and the assumption of a family of exponentially decaying autocorrelation functions. The purpose of this paper is to interpret these observations by developing the complex amplitude autocorrelation function when both incoherent and coherent backscatter are present. Using this expression, it is then shown that when coherent scatter is present, the temporal coherency increases as observed. Data are analyzed in snow and in rain. The results agree with the theoretical expectations, and the authors interpret this agreement as an indication that coherent scatter is the likely explanation for the observed enhanced temporal coherency. This finding does not affect decorrelation times measured using time series. However, when the time series is not available (as in theoretical studies), the times to decorrelation are often computed based upon the assumptions that the autocorrelation function is a member of the family of exponentially decaying autocorrelation functions and that the signal decorrelation is due solely to the Doppler velocity fluctuations associated with incoherent scatter. Such an approach, at times, may significantly underestimate the true required times to decorrelation thus leading to overestimates of statistical reliability of parameters.
\end{abstract}

\section{Introduction}

The autocorrelation function (AC) of complex amplitudes is well established in radar meteorology for incoherent scatter. Regardless of the form of AC, when one looks at the products of complex amplitudes, whether it be for calculating the backscattered intensities or the complex amplitude autocorrelation functions, time averaging has always been used to argue that the fluctuating components of each arising from differential particle velocities all averaged to naught (e.g., Lhermitte 1960; Atlas 1964, p. 396; Sauvageot 1992, p. 50). While a few investigators recognized that some particles could be moving at the same velocity (e.g., Sauvageot 1992, p. 50), it was then usually argued that because of their random spatial positions these equal velocity particles would make no net contribution to the average backscattered power. Indeed it can be shown theoretically that this is true

Corresponding author address: Dr. Arthur R. Jameson, 5625 N. 32nd St., Arlington, VA 22207-1560.

E-mail: arjatrjhsci@verizon.net when there is no spatial correlation on any scale among the scatterers over a large domain (a near impossibility in the atmosphere), but it is not true when there are spatial correlations of the structures of the precipitation on scales of the radar wavelength. The existence of candidate structures is readily apparent in the passage of backlit snow and as waves of rain sweeping across the pavement. If the scales of these structures are in resonance with the wavelength, then coherent backscatter can occur (Jameson and Kostinski 2010).

While the purpose of Jameson and Kostinski (2010) was to report the apparent existence of radar coherent backscatter from precipitation, the primary purpose of this paper is to develop theoretical expressions for the complex amplitude autocorrelation function when both incoherent and coherent backscatter are present, which was not done in the previous work. We then show how the presence of coherent scatter leads to the enhanced temporal coherence of the signals as actually observed in data.

To that end, we compare the results of the theory with the set of observations used by Jameson and Kostinski 
(2010). The radar data in both rain and snow were collected using the National Science Foundation Colorado State University-University of Chicago-Illinois State Water Survey (CSU-CHILL) Radar Facility at Greeley, Colorado. This radar has a $1.1^{\circ}$ beamwidth. It operates at a frequency of $2.725 \mathrm{GHz}$ corresponding to a nominal wavelength of $11.01 \mathrm{~cm}$. While the antenna was held stationary, time series observations of the backscattered complex amplitudes ( $I, Q$ pairs, where $I$ and $Q$ indicate the real and imaginary parts, respectively) were collected 1024 times a second at vertical polarization. In the rain, observations were collected over 332 bins of $150-\mathrm{m}$ range over a distance of about $3-53 \mathrm{~km}$ from the radar. The elevation angle was $1.82^{\circ}$ so the bottom of the main lobe of the beam was around $600 \mathrm{~m}$ above the surface at about $30-\mathrm{km}$ range. These measurements are through weak convection containing a few convective cores. Likewise, observations were gathered in snow over 218 bins of 150-m range over a distance of about $3.30-36 \mathrm{~km}$ from the radar. The elevation angle was $2.54^{\circ}$ so the bottom of the main lobe of the beam was around $700 \mathrm{~m}$ above the surface at about range $20 \mathrm{~km}$.

Before discussing these data further, however, we first look at the theory for an autocorrelation function when both incoherent and coherent backscatter is occurring.

\section{Theory}

The net electric field at a location produced by spatially distributed scatterers can be expressed as

$$
E(t)=\sum_{i} a_{i} e^{j\left(\omega_{i} t-2 \mathbf{k} \cdot \mathbf{r}_{i}\right)},
$$

where $a_{i}$ is the amplitude of the field scattered by the $i$ th particle at location $\mathbf{r}_{i}$ from the observer, $\omega_{i}$ is its Doppler angular frequency, and $\mathbf{k}$ is the wavenumber along the direction of propagation and the factor of 2 accounts for a round trip. Similarly at time $(-\tau)$, where $\tau$ is a lag time, we have that

$$
E(t-\tau)=\sum_{m} a_{m} e^{j\left[\omega_{m}(t-\tau)-2 \mathbf{k} \cdot \mathbf{r}_{m}\right]} .
$$

It follows, then, that

$$
\begin{aligned}
\left\langle E^{*}(t) E(t-\tau)\right\rangle= & \left\langle\sum_{i} a_{i}^{2} \exp \left(-j \omega_{i} \tau\right)\right\rangle \\
& +\left\langle\sum_{i \neq} \sum_{m} a_{i} a_{m} \exp \left[j \omega_{i} t-\omega_{m}(t-\tau)\right]\right. \\
& \left.\times \exp \left[j 2 \mathbf{k} \cdot\left(\mathbf{r}_{i}-\mathbf{r}_{m}\right)\right]\right\rangle
\end{aligned}
$$

where $\langle\cdot\rangle$ represents the time average over the observation interval. It is shown in appendix A in Jameson and Kostinski (2010) that coherent scatter is possible when $\omega_{i}=\omega_{m}$. Consequently, the second term in (3) can be separated into two components; namely,

$$
\begin{aligned}
\left\langle E^{*}(t) \times E(t-\tau)\right\rangle= & \left\langle\sum_{i} a_{i}^{2} \exp \left(-j \omega_{i} \tau\right)\right\rangle+\left\langle\sum_{i \neq} \sum_{m} a_{i} a_{m} \exp (-j \tau \omega) \times \exp \left[j 2 \mathbf{k} \cdot\left(\mathbf{r}_{i}-\mathbf{r}_{m}\right)\right]\right\rangle_{\omega_{i}=\omega_{m}=\omega_{o}} \\
& +\left\langle\sum_{i \neq} \sum_{m} a_{i} a_{m} \exp \left[j\left(\omega_{i}-\omega_{m}\right) t\right] \times \exp \left[j 2 \mathbf{k} \cdot\left(\mathbf{r}_{i}-\mathbf{r}_{m}\right) \times \exp \left(-\tau \omega_{m}\right)\right]\right\rangle_{\omega_{i} \neq \omega_{m}}
\end{aligned}
$$

The time average of the last term in (4) approaches the null after sufficient averaging ( $\sim 50-100 \mathrm{~ms})$, and we are then left with

$$
\begin{aligned}
\left\langle E^{*}(t) \times E(t-\tau)\right\rangle= & \left\langle\sum_{i} a_{i}^{2} \exp \left(-j \omega_{i} \tau\right)\right\rangle \\
& +\left\langle\sum_{i \neq} \sum_{m} a_{i} a_{m} \exp \left[j 2 \mathbf{k} \cdot\left(\mathbf{r}_{i}-\mathbf{r}_{m}\right)\right]\right. \\
& \left.\times \exp (-j \tau \omega) \delta\left(\omega-\omega_{0}\right)\right\rangle \\
= & \langle\mathrm{AC}(\tau)\rangle
\end{aligned}
$$

where $\mathrm{AC}$ is the complex amplitude autocorrelation function. Since the average complex amplitude is null, the mean values that normally appear in the definition of an autocorrelation function do not appear in (5). The $\omega_{\mathrm{o}}$ correspond to the Doppler velocity of the different grids of scatterers associated with coherent scatter so the Kronecker delta function simply denotes that it is only those frequencies that contribute to (5). In contrast to purely incoherent scatter, coherent scatter introduces a second term into AC. The first term in (5) corresponds to the incoherent scatter component of the autocorrelation function (its Fourier transform is the incoherent scatter component of the Doppler spectrum). The second term corresponds to the coherent scatter component of autocorrelation function (its Fourier transform is the coherent scatter component of the Doppler spectrum). 
Ignoring the small changes in particle radar cross sections arising from oscillations or tumbling, (5) can be simplified even further since the $a_{i}$ s are then independent of $\tau$. While the $\left(\mathbf{r}_{i}-\mathbf{r}_{m}\right)$ s are also independent of $\tau$, they may still depend weakly on time because the $\omega$ s of particles within the clusters may vary slightly from the mean $\omega_{0}$. Hence, using the results in Jameson and Kostinski (2010, appendix A), we have that

$$
\begin{aligned}
\langle A C(\tau)\rangle= & \left\langle\sum_{i} a_{i}^{2} \exp \left(-j \omega_{i} \tau\right)\right\rangle \\
& +\sum_{\omega_{0}}\left[\left\langle\sum_{i \neq} \sum_{m} a_{i} a_{m} \exp \left[j 2 \mathbf{k} \cdot\left(\mathbf{r}_{i}-\mathbf{r}_{m}\right)\right]\right\rangle\right. \\
& \left.\times\left\langle\exp (-j \omega \tau) \delta\left(\omega-\omega_{0}\right)\right\rangle\right] \\
= & \left\langle\sum_{i} a_{i}^{2} \exp \left(-j \omega_{i} \tau\right)\right\rangle \\
& +\sum_{\omega_{0}}\left\langle I_{C}\left(t, \omega_{0}\right)_{\text {coherent }} \exp \left(-j \omega_{0} \tau\right)\right\rangle \\
= & \langle\operatorname{AC}(\tau)\rangle_{\text {Incoherent }}+\langle\operatorname{AC}(\tau)\rangle_{\text {Coherent }}
\end{aligned}
$$

where the $\langle\cdot\rangle$ indicate time average, and the summation is now over velocities $\omega_{0}$ and $I_{\mathrm{C}}$ is the intensity of the coherent backscatter given by Eq. (A16) in Jameson and Kostinski (2010). Because all of these quantities are complex numbers, by the triangle inequality, the magnitude of the left-hand side is less than or equal to the sum of the magnitudes of each of the terms on the righthand side of (6). However, the minimum contribution that coherent scatter can make occurs when it is orthogonal to the incoherent component in the complex plane. Hence, at the very least we have

$$
\frac{|\langle\mathrm{AC}(\tau)\rangle|}{\left.\langle\operatorname{AC}(\tau)\rangle\right|_{\text {Incoherent }}}=1+\frac{|\mathrm{AC}(\tau)|_{\text {Coherent }}}{|\langle\mathrm{AC}(\tau)\rangle|_{\text {Incoherent }}} .
$$

Consequently, when coherent scatter is present the magnitude of the autocorrelation function is enhanced beyond what it would have been if only incoherent scatter were present for any $\tau$. However, this is somewhat misleading because what we are really interested in are the magnitudes $\rho$ of the autocorrelation functions normalized by the total power at $\tau=0$.

Rewriting (7) we then have

$$
\begin{aligned}
\frac{\left(I_{0}+I_{C}\right) \rho}{I_{0} \rho_{\text {Incoherent }}} & =1+\frac{I_{C} \rho_{\text {Coherent }}}{I_{0} \rho_{\text {Incoherent }}}, \text { so } \\
\frac{\rho}{\rho_{\text {Incoherent }}} & =\frac{I_{0}}{\left(I_{0}+I_{C}\right)}+\frac{I_{C} \rho_{\text {Coherent }}}{\left(I_{0}+I_{C}\right) \rho_{\text {Incoherent }}},
\end{aligned}
$$

or more simply

$$
\rho=(1-\mathfrak{F}) \rho_{\text {Incoherent }}+\mathfrak{F} \rho_{\text {Coherent }},
$$

where $\mathfrak{F}=I_{\mathrm{C}} /\left(I_{\mathrm{C}}+I_{0}\right)$ is the fractional contribution of the coherent power to the total power, while $I_{0}$ and $I_{\mathrm{C}}$ refer to the incoherent and coherent backscattered intensities, respectively.

Now let us consider examples for which $\mathfrak{F}$ is fixed but the $\rho$ s change. Suppose there is a "coherent target" such as an airplane embedded within precipitation (see Figs. $1 \mathrm{a}, \mathrm{b})$. That is, the precipitation can reshuffle but the parts of the aircraft do not, much to the relief of the passengers. (Actually, the aircraft can "decorrelate," for example, in that its radar cross section can change in time, but, as Fig. 1b illustrates, that is usually a slow process, depending upon the radar perspective.) Now if $\rho_{\text {Coherent }}=\rho_{\text {Incoherent }}$, then $\rho=\rho_{\text {Incoherent }}$ so there would be no difference from pure incoherent scatter in conflict with our suppositions that we have added coherent scatter and that the passengers are not in real trouble. That is, everything would reshuffle as though no aircraft had been added, which is clearly not the case in Fig. $1 \mathrm{~b}$. If $\rho_{\text {Coherent }}<\rho_{\text {Incoherent }}$ then $\rho<\rho_{\text {Incoherent }}$, and it would mean that somehow the addition of the aircraft actually enhanced decorrelation in conflict with the physics and with observations of aircraft embedded within precipitation (Fig. 1b). The remaining possibility is that $\rho_{\text {Coherent }}>\rho_{\text {Incoherent }}$; so $\rho>\rho_{\text {Incoherent }}$ when a coherent target is present as observed in the case of an aircraft. We expect the same thing to happen when there are grids of particles producing coherent scatter that do not reshuffle as rapidly as the incoherent scatterers so the time it takes to decorrelate to any level will increase when coherent scatter is present. This is illustrated in Fig. 1c using (9) where the incoherent component is calculated after a partial filtering of the low-frequency components of the reflectivity power spectrum $Z(f)$, as discussed in Jameson and Kostinski (2010, appendix B). That is (and without going into details here), partial filtering means that the spectral power attributable to coherent scatter has been removed from those $f$ associated with the coherent scatter and $\rho$ is then recomputed for this filtered time series. [The ratio $I_{C} /\left(I_{0}+I_{C}\right)$ is estimated using $Z(f)$ as discussed in Jameson and Kostinski (2010).] Unlike an aircraft, however, the particle grids thought to be responsible for the coherent scatter come and go, so $\rho$ varies more than for an aircraft as Fig. 1c illustrates.

\section{Some observations}

Using the data described above, the 1000-point Doppler spectra were calculated and then used to compute the 

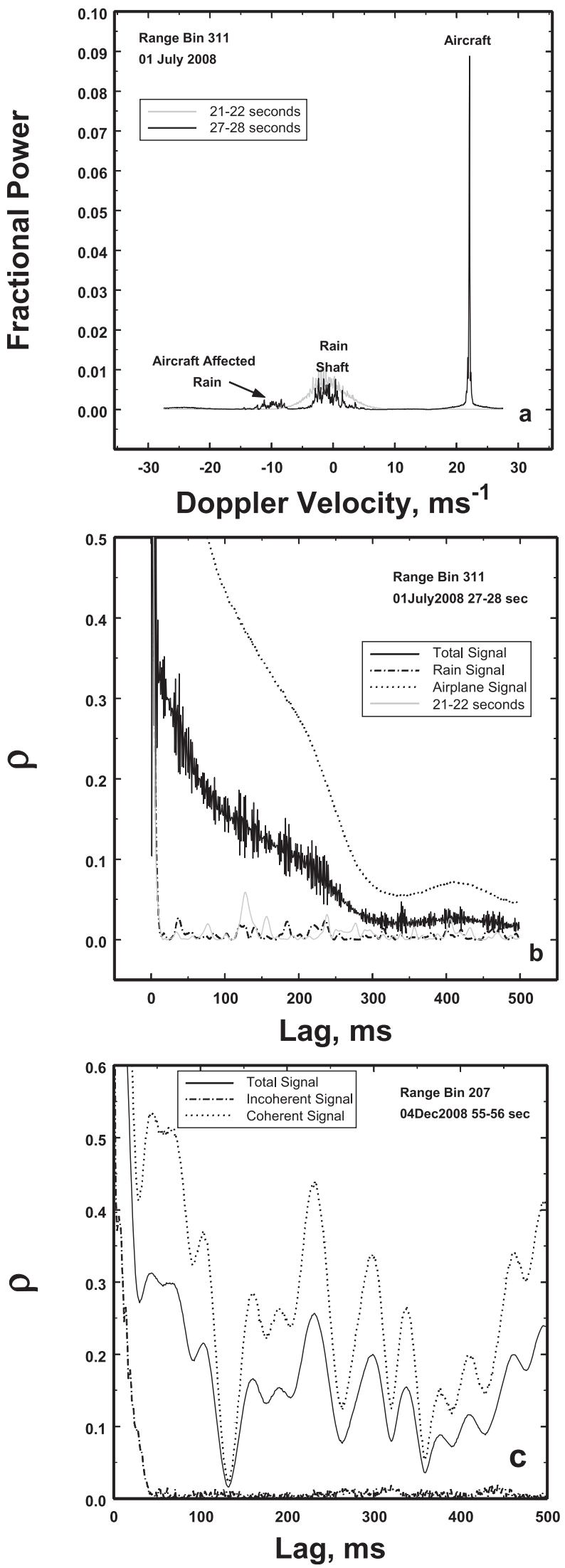

observed power-weighted standard deviations of the velocities $\sigma_{V}$, as can be found in many references in radar meteorology including Doviak and Zrnić (1993, p. 135) and Sauvageot (1992, p. 183). These, in turn, were used to calculate the expected 1/e times to decorrelation (where $e$ is Euler's number) using the standard deviation of the velocities assuming that the Doppler spectra have exponentially decaying autocorrelation functions (discussed below and in the appendix). [The decorrelation time is simply the time (pulse to pulse lags) it takes for the complex amplitudes-powers to become statistically independent because of particle relative motions.] That is, using the relation (6.24) in Atlas (1964, and many other references), but corrected for a missing factor of $\sqrt{2}$ as discussed in the appendix, it is easy to show that

$$
\tau_{1 / e}=\frac{1.125 \lambda}{\sigma_{V}},
$$

where $\lambda$ is the radar wavelength in centimeters, $\sigma_{V}$ is in meters per second, and $\tau$ is in milliseconds. Note that the directly measured decorrelation times for the amplitudes include the decorrelation due to noise. That same noise decorrelation appears in the Doppler spectra as expressed through $\sigma_{V}$ in (10), and it must be included for a fair comparison (described below) of the two sets of $\tau_{1 / e}$.

The reason for using the 1/e time to decorrelation is that, unlike the time to 0.01 decorrelation, it is relatively easy to measure directly [independent of (10)] from the autocorrelation function $(\rho)$ of the magnitudes of the complex amplitudes when it drops below 1/e. For incoherent scatter and in so far as the Doppler spectra were associated with exponentially decaying $\rho$, the values for $\tau_{1 / e}$ computed from the Doppler velocity standard deviations and those directly measured using $\rho$ should be quite similar. That is not what Jameson and Kostinski (2010) found as repeated below but corrected for the factor of $\sqrt{2}$.

With a range of the observed standard deviations of the Doppler velocities on the order of up to a few meters per second in both the snow and the rain, one would

FIG. 1. (a) Doppler velocity spectra before an aircraft penetrated the radar sample volume (21-22 s) and while an aircraft was present (27-28 s). (b) The magnitudes of the complex autocorrelation function $\rho$ corresponding to the two cases in (a) as well as the recalculated $\rho$ corresponding to the incoherent rain signal after removal of the airplane signal. Note the similarity between the prior aircraft and recomputed $\rho$. (c) The $\rho$ for the total coherent plus incoherent signals in snow, the estimated $\rho$ corresponding to recalculated incoherent component, and that for the coherent component derived using (8). The contrast with an airplane is clear. 
expect the usual 1/e decorrelation times of around $5-11 \mathrm{~ms}$ at the most. While $5-6 \mathrm{~ms}$ is the mean in Fig. 2 observed directly in the rain, the peak calculated using $\sigma_{V}$ is only $4 \mathrm{~ms}$ ( $76 \%$ of the calculated $\tau \mathrm{s}$ are $\left.\leq 5 \mathrm{~ms}\right)$. Moreover, $45 \%$ of the observed values are larger than $5 \mathrm{~ms}$ with $5 \%$ of the values $\geq 10 \mathrm{~ms}$. The snow is even more remarkable with a peak in the histogram frequency (Fig. 2b) of the directly observed values of $20 \mathrm{~ms}$ and a mean of about $21 \mathrm{~ms}$, yet the mean value derived using $\sigma_{V}$ is only $5.6 \mathrm{~ms}(74 \%$ of the calculated $\tau$ are $\leq 6 \mathrm{~ms}$ ). Furthermore, $30 \%$ of the observed values occur at $\tau \geq 25 \mathrm{~ms}$. These $\tau$ are significantly greater than one would expect for the traditional, incoherent scatter decorrelation. For example, in the snow at range bin 131 between 28 and $29 \mathrm{~s}$, the observed standard deviation of the velocity was $1.50 \mathrm{~m} \mathrm{~s}^{-1}$ [over the velocity domain of -7.022 to $0.000 \mathrm{~m} \mathrm{~s}^{-1}$; for a plot of this spectrum, see Fig. B1 in Jameson and Kostinski (2010)]. According to classical theory [as modified in (10)], this implies $1 / e$ decorrelation time of about $8 \mathrm{~ms}$, yet the observed value was $16 \mathrm{~ms}$. Clearly, the observed large values cannot be used as a measure of the time to decorrelation for the incoherent component. The $\sigma_{V}$ is the power-weighted value computed from the Doppler spectrum as discussed earlier, while the $\tau_{1 / e}$ is computed directly from the magnitude $(\rho)$ of the complex autocorrelation function.

There are two possibilities for this discrepancy. One obviously is that $\rho$ need not be exponentially decaying. Indeed, that is, in part, the case as we show next by considering the generalized exponential (e.g., Kostinski and Koivunen 2000) given by

$$
\rho(\tau)=\exp \left(-C \tau^{\alpha}\right)
$$

where $C$ and $\alpha$ are constants, and $\tau$ is the lag time. For example, for a Gaussian autocorrelation function, $\alpha=2$; while for a simple exponential, $\alpha=1$. Kostinski and Koivunen (2000) show that, when $\alpha=2$, the samplecovariance matrix is ill conditioned so for real data $\alpha$ should be $<2$. While inspections of the $\rho$ in rain and snow all show an "exponential like" decay at the smaller lags, it is often followed by much slower or, at times, even increasing $\rho$ at longer lags, particularly in snow.

To understand what is happening, we estimate $\alpha$ from observations using the relation

$$
\ln \{|\ln [\rho(\tau)]|\}=\ln (C)+\alpha \ln (\tau) .
$$

Two examples, one from snow and the other from rain, are shown in Fig. 3. In both cases there are two components of $\rho$ just as in (6). The first is over domains in $\tau$ where the $\rho$ seem to be represented by (11). The second components we attribute to nonvelocity intensity oscillations.

In the snow example, the observed slopes were $\alpha=$ 1.70 for $3 \leq \tau \leq 20 \mathrm{~ms}$, while in rain $\alpha=1.908$ for $1 \leq$ $\tau \leq 10 \mathrm{~ms}$. These are clearly not Gaussian and only partially exponentially decaying. What else does this figure show? The dash-dot line corresponds to an estimate of what $\rho$ would have been had it been purely incoherent. [This estimate is the $\rho$ recomputed after partial filtering of the lower frequencies of $Z(f)$, as discussed above.] As the zero ordinate $(y)$ line shows in Fig. 3, apparently, only when the coherent scatter is present do the $\alpha$ decrease and the $\tau_{1 / e}$ increase [i.e., the $\ln (\tau)_{y=0}$ is larger], in these observations. Hence, we attribute the increase in decorrelation times to the presence of coherent scatter. [These findings are also consistent with our observation that partial filtering of the lower frequencies in $Z(f)$ associated with apparent coherent scatter significantly reduces the time to decorrelation, e.g., Fig. 1b in this paper and Fig. B1c in Jameson and Kostinski (2010).]

Actually, the results are even stronger than Fig. 3 implies. To show this, we note that the decorrelation to some level $\mathfrak{L}$ at lag $\tau>0$ requires a phase difference of $\Delta \phi(\tau)$. Regardless of $\alpha$, however, all decreasing exponentials, such as those in the family defined by (11), require that $\Delta \phi(\tau)=1 \mathrm{rad}$ to reach the 1/e decorrelation. But what do the data show?

As mentioned above, we directly observe the standard deviation of the Doppler velocity spectrum $\sigma_{V}$ and the 1/e time to decorrelation of the lag $\tau_{1 / e}$, which is the lag at which the observed $\rho$ drops below 0.3679. From those two observations, it is possible to estimate the rms phase change $\Delta \phi_{1 / e}$. This provides a measure of the amount of particle reshuffling with respect to the wavelength required to achieve the 1/e decorrelation as discussed in the appendix. Specifically, (A10) can be written as

$$
\Delta \phi_{1 / e}=\frac{4 \pi}{\lambda}\left(\frac{\tau_{1 / e} \sigma_{v}}{\sqrt{2}}\right)
$$

where the variables have all been previously defined and the quantity in parentheses is the rms reshuffling distance. In Fig. 4 the values of $\Delta \phi_{1 / e}$ corresponding to the 1 minute of snow and 1 minute of rain data (Jameson and Kostinski 2010) are presented. The differences between rain and snow are obvious. In particular, in the snow almost all cases the $\Delta \phi_{1 / e}$ are greater, sometimes far greater, than the value required for the family of exponentially decaying autocorrelation functions denoted by the dashed line. In the rain the same is true, but the values are generally smaller than for the snow and many more of them lie near 1 rad. In general, though, in both 

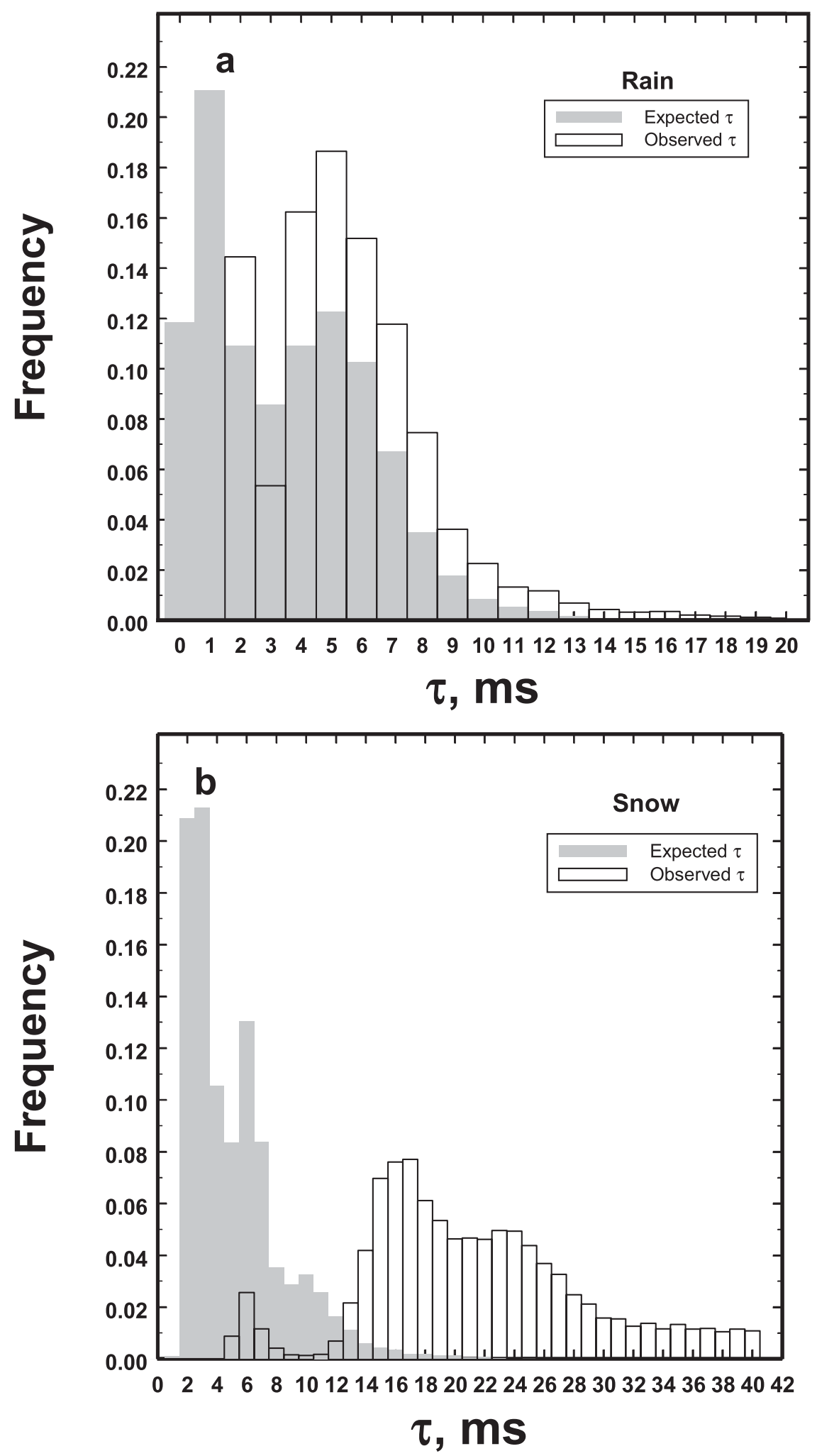

FIG. 2. The histograms of the 1/e times to decorrelation for (a) 15600 samples in the rain and (b) 10400 samples in the snow. The expected $\tau$ are calculated using the observed standard deviations of the Doppler velocities and the new expression (10). The excess observed correlation in both rain and snow indicates the presence of an additional source of coherence. 

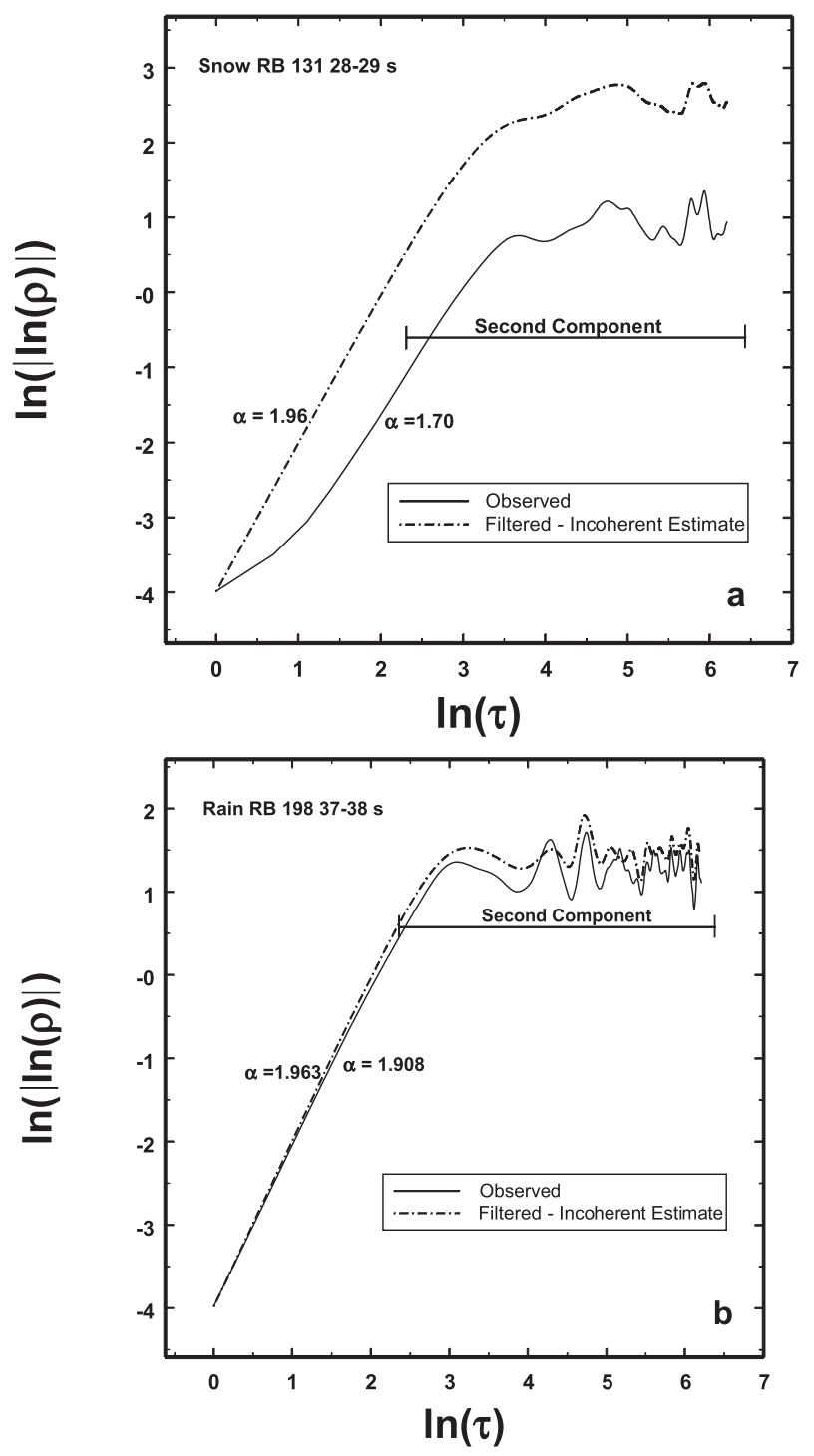

FIG. 3. Plots for determining $\alpha$ for an autocorrelation function of the form $\rho(\tau)=\exp \left(-C \tau^{\alpha}\right)$ in (a) snow and (b) rain. Note that $\rho$ has two components and that the part associated with a constant $\alpha$ is connected to the incoherent scatter component as discussed in the text.

cases the decorrelation is slower, often much slower, than can be described using a family of decaying exponentials. While some of this increase likely reflects the influence of noise, such noise should be unbiased and small so the clear excess beyond unity is likely real. (For 1000-point FFTs, $\sigma_{V}$ should be known to within better than $\pm 5 \%$ while $\tau_{1 / e}$ is likely known to within $\pm 15 \%$ in agreement with the thickness of the band near the bottom of Fig. 4b.) Because of the substantial preponderance of nonexponential behavior, it is unlikely that they can be explained assuming the usual models of velocity fluctuation spectra associated with incoherent scatter. Rather, as in Fig. 3, it is likely that the second component of $\rho$ associated with the coherent backscatter is responsible for the enhanced temporal coherency expressed by the enhanced $\Delta \phi_{1 / e}$. These conclusions are consistent with the theory in the previous section. We discuss this further below.

\section{Discussion}

For classic incoherent scatter it turns out that the time to decorrelation is nothing but the consequence of the power weighting of the differential frequencies $f$. That is, if we just consider incoherent scatter for the moment in (6) and if we drop the brackets, the magnitude of the incoherent autocorrelation function is given by

$$
\begin{aligned}
\rho(\tau) & =\left\{\frac{\sum_{i} a_{i}^{4}}{\left(\sum_{i} a_{i}^{2}\right)^{2}}+\sum_{i \neq} \sum_{j} \frac{a_{i}^{2} a_{j}^{2} \cos \left[\left(\omega_{i}-\omega_{j}\right) \tau\right]}{\left(\sum_{i} a_{i}^{2}\right)^{2}}\right\}^{1 / 2} \\
& =\left\{\frac{\sum_{i} a_{i}^{4}}{\left(\sum_{i} a_{i}^{2}\right)^{2}}+\sum_{i \neq} \sum_{j} \frac{a_{i}^{2} a_{j}^{2} \cos [(4 \pi f \tau)]}{\left(\sum_{i} a_{i}^{2}\right)^{2}}\right\}^{1 / 2} .
\end{aligned}
$$

Using this form of $\rho$, it is the second term under the square root that determines the temporal characteristics of the autocorrelation function and represents the power weighting by the differential frequencies, $f$ as has been known for years (e.g., Atlas 1964 and references therein). As a simple example, a Gaussian Doppler velocity spectrum is plotted in Fig. 5 along with the corresponding $\rho^{2}$ calculated using (13). The Fourier transform of that $\rho^{2}$ yields the so-called fluctuation spectrum arising from the velocity differences among the particles (e.g., see Atlas 1964). But do these fluctuations explain all of the temporal decorrelation? We believe not.

For lower frequencies, the phases among the particles take longer to change over time. Thus, as calculations using (13) confirm, the more the smaller differential frequencies are weighted, the longer the decorrelation times. This makes physical sense because $\Delta \phi$ increases with increasing $\tau$ and increasing differential frequencies $f$. The narrower the Doppler spectra, the more the smaller $f$ are weighted, so it takes more time $(\tau)$ for $\Delta \phi$ to reach levels for significant decorrelation. That is, the time to decorrelation is longer. For broad Doppler spectra, the opposite holds true because the larger $f$ contribute more, so it takes less time $(\tau)$ to decorrelate because the phase changes more rapidly. Thus, for any peaked Doppler power spectrum, the small differential $f$ are going to the 

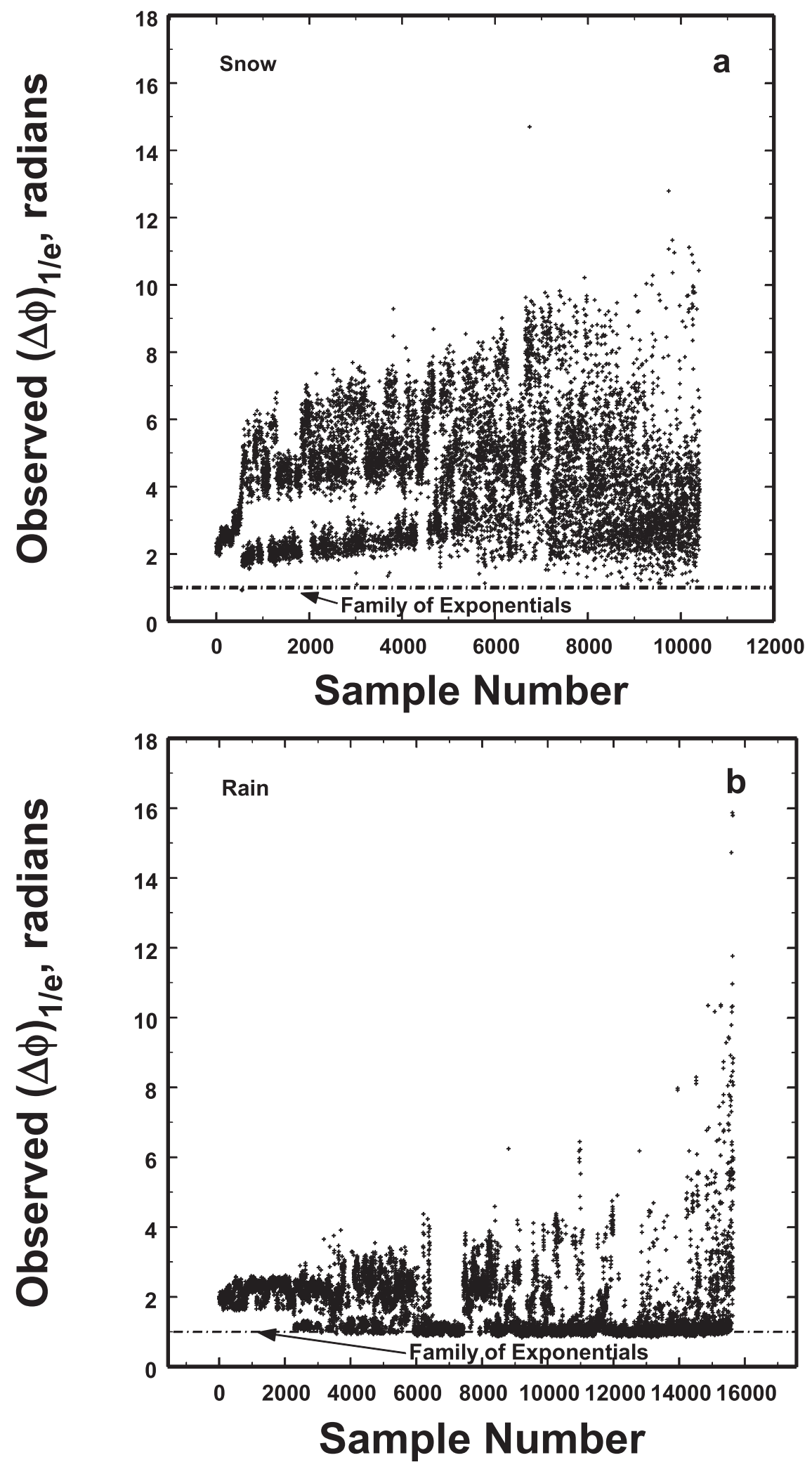

FIG. 4. Plots of the change in phase $\Delta \phi_{1 / e}$ in the autocorrelation function $\rho$ required to achieve decorrelation to the 1/e level: (a) snow and (b) rain. For the family of decaying exponentials, $\Delta \phi_{1 / e}=1 \mathrm{rad}$. The excess $\Delta \phi_{1 / e}$ in the observations most likely arises from the second component of $\rho$, associated with coherent scatter. 

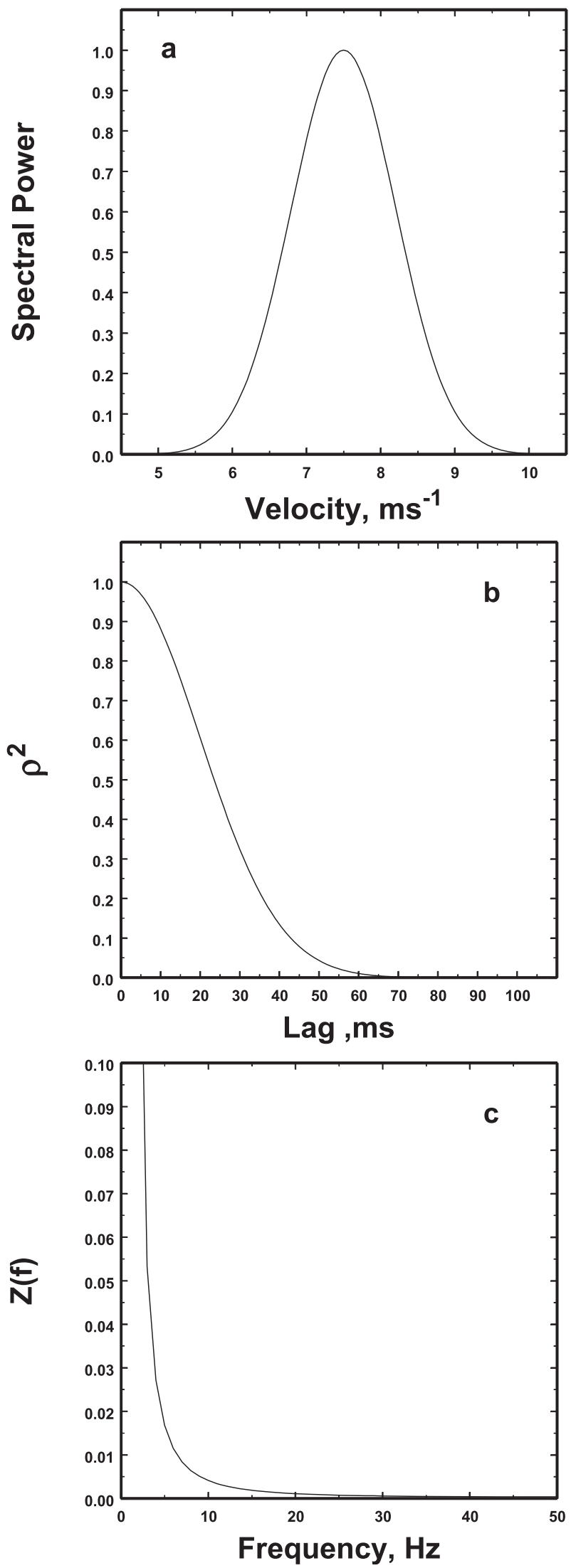

ones most heavily power weighted thus producing a $\rho$ peaked at zero.

However, that is not what is causing the enhanced temporal coherence. We note that the Fourier transform of (6b) yields a Doppler velocity spectrum of the incoherent part plus a sum of $\delta\left(\omega-\omega_{0}\right)$ spikes (where $\delta$ is the Kronecker delta) corresponding to the coherent backscatter components moving at their respective velocities $\omega_{0}$ (Jameson and Kostinski 2010). These are the statistically meaningful spikes in excess of those expected for the Erlang probability distribution function (pdf) (associated with the Rayleigh signal statistics of measurements using a stationary antenna) sometimes observed in real Doppler spectra (e.g., Jameson and Kostinski 2010, their Fig. B3). Because they are so narrow they contribute to the weighting function (fluctuation spectrum) at the smaller differential frequencies along with those associated with incoherent scatter. However, it is important to note that it is not these Doppler velocity spikes that are responsible for the observed spectral powers of $Z(f)$ at lower frequencies as has been verified by removing them from the Doppler spectra and then recalculating $Z(f)$. Rather, as Jameson and Kostinski (2010) show (e.g., see their Fig. 3), it is the non-Doppler velocity oscillations in $Z(f)$ hypothesized to be induced by the motion of the coherent scatter grids themselves that strongly power the lower frequencies of $Z(f)$ well beyond the spectral power contribution of any velocity fluctuations and that produce the extended times to decorrelation. As reflected in wavelet analyses (Jameson 2010) as modulations of wavelet strength, these fluctuations in $Z$ appear as oscillations of the intensity of those Doppler spectral spikes associated with the coherent scatter.

This may, at times, have important implications regarding the theoretical treatment of errors in radar meteorology that are all based upon the assumption that the signal decorrelation is due solely to the Doppler velocity fluctuations associated with incoherent scatter. Given the apparent frequency of occurrence of coherent scatter (Jameson and Kostinski 2010, their Fig. 7a), it is likely that such calculations may, at times, significantly underestimate the true required times to decorrelation and, hence, to overestimates of statistical reliability of the measurements. That is, if one is able to compute the decorrelation time directly from the time series, the classical signal statistics are correct since the sources of

FIG. 5. (a) A plot of a Gaussian Doppler spectrum, (b) $\rho^{2}$ calculated using (13) corresponding to the spectrum in (a), and (c) the fluctuation spectrum corresponding to (b). 
the coherent scatter act like "big drops" moving in the wind like any other scatterer. This is true regardless of whether the antenna is stationary or moving [although moving antennas causes the statistics to be non-Rayleigh (Jameson and Kostinski 1996, 2008; Jameson 2008); while a moving antenna essentially makes it impossible to detect the coherent scatter, it is still there]. There is no separable coherent scatter effect on $\tau_{1 / e}$ although coherent scatter may still affect the power observations. If, however, one is performing theoretical calculations or one only has the variances of Doppler velocities from some real data but no access to the time series, inferences of the times to decorrelation and the number of independent samples will likely be in error. Figure 1 suggests that in snow the number of independent samples would be a factor of 4 or 5 smaller than one would have estimated while in rain, such estimates could be smaller by a factor of 3 or more. However, we emphasize that the most important development in this work is (6) and the questions that have been raised. Hopefully, whatever follows as a consequence will be enlightening.

Acknowledgments. This work was supported by the National Science Foundation (NSF) under Grants ATM05-31996 (AJ) and ATM08-04440 as well as ATM0554670 (AK). We gratefully acknowledge the meticulous data acquisition by Dave Brunkow and Pat Kennedy of the NSF CSU-CHILL National Radar Facility operated by the Colorado State University (CSU).

\section{APPENDIX}

\section{The Variance of the Fluctuation Spectrum and the rms Fluctuation Velocity}

The remark that the variance of the fluctuation spectrum is twice that of the associated Doppler velocity spectrum is stated in the readily available literature without any accompanying derivation. Likewise the discussion of the differential phase associated with the decay of the autocorrelation function is also confusing and often vague. The purpose of this brief appendix is to present a concise development of these two concepts.

For the Doppler spectral velocities $v$, we have that

$$
\begin{gathered}
\Delta v_{i}=v_{i}-\bar{v} \text { and } \Delta v_{j}=v_{j}-\bar{v} . \\
\text { Hence, } v_{i}=\Delta v_{i}+\bar{v} \text { and } v_{j}=\Delta v_{j}+\bar{v}, \text { so } \\
v_{i}-v_{j}=\Delta v_{i}-\Delta v_{j} .
\end{gathered}
$$

Let $V=v_{i}-v_{j}$ be the fluctuation velocity. Remembering that for every $+V$ there is a $-V$ we know that the fluctuation spectrum must be symmetric about zero with mean zero. The variance of $V$ is then given by

$$
\begin{aligned}
\overline{(v-\bar{v})^{2}} & =\overline{\sum_{i} \sum_{j}\left(v_{i}-v_{j}\right)^{2}} \\
& =\overline{\sum_{i} \sum_{j}\left(\Delta v_{i}^{2}+\Delta v_{j}^{2}\right)-2 \Delta v_{i} \Delta v_{j} .}
\end{aligned}
$$

Since the is and js are independent we can rewrite this to be

$$
\begin{aligned}
\sigma_{V}^{2}=\overline{\sum_{i} \Delta v_{i}^{2}} & +\overline{\sum_{j} \Delta v_{j}^{2}}-2 \overline{\sum_{i} \sum_{j}\left(v_{j}-\bar{v}\right)\left(v_{j}-\bar{v}\right)} \\
=\sigma_{v}^{2}+\sigma_{v}^{2} & -2 \overline{\sum_{i} \sum_{j}\left(v_{i}-\bar{v}\right)\left(v_{j}-\bar{v}\right)} \\
\operatorname{But} \overline{\sum_{i} \sum_{j} \Delta v_{i} \Delta v_{j}} & =\overline{\sum_{i} \sum_{j}\left(v_{i}-\bar{v}\right)\left(v_{j}-\bar{v}\right)} \\
& =\sum_{i} \sum_{j}\left(\overline{v_{i} v_{j}}-\overline{v v_{j}}-\overline{v v_{i}}+\bar{v}^{2}\right) \\
& =\sum_{i} \sum_{j}\left(\bar{v}^{2}-\bar{v}^{2}-\bar{v}^{2}+\bar{v}^{2}\right)=0, \\
\sigma_{V}^{2} & =2 \sigma_{v}^{2}
\end{aligned}
$$

Hence, the variance of the fluctuation spectrum is, indeed, twice the variance of the Doppler spectrum regardless of the form of that spectrum. Furthermore, since the average $V$ is zero, we have

$$
\overline{V^{2}}=2 \sigma_{v}^{2} \text { so that } \sqrt{\overline{V^{2}}}=\sqrt{2} \sigma_{v} .
$$

Hence, the rms phase shift at lag $\tau$ is simply

$$
\Delta \phi(\tau)=\frac{\sqrt{2} \sigma_{v} 2 \pi \tau}{\lambda}
$$

or, in a form more compatible with past expressions,

$$
\Delta \phi(\tau)=\frac{4 \pi \sigma_{v} \tau}{\lambda \sqrt{2}}
$$

In particular, then, when $\tau$ is the $1 / e$ decorrelation time of the magnitude of the autocorrelation function, it follows that

$$
\begin{aligned}
\Delta \phi_{1 / e} & =\frac{4 \pi \sigma_{v} \tau_{1 / e}}{\lambda \sqrt{2}}, \text { and } \\
\tau_{1 / e} & =\frac{\lambda \sqrt{2} \Delta \phi_{1 / e}}{4 \pi \sigma_{v}}
\end{aligned}
$$


for any Doppler spectrum. For those having an exponentially decaying autocorrelation function, $\Delta \phi_{1 / e}=1$.

\section{REFERENCES}

Atlas, D., 1964: Advances in radar meteorology. Advances in Geophysics, Vol. 10, Academic Press, 317-478.

Doviak, R. J., and D. S. Zrnić, 1993: Doppler Radar and Weather Observations. 2nd ed. Academic Press, 562 pp.

Jameson, A. R., 2008: Radar observations of rainfall variability using non-Rayleigh signal fluctuations. J. Appl. Meteor. Climatol., 47, 607-619.

2010: On the temporal characteristics of radar coherent structures in snow and rain. J. Appl. Meteor. Climatol., 49, in press.
_ , and A. B. Kostinski, 1996: Non-Rayleigh signal statistics caused by relative motion during measurement. J. Appl. Meteor., 35, 1846-1859.

— and - 2008: The effect of clustering on the uncertainty of differential reflectivity measurements. J. Appl. Meteor. Climatol., 47, 2816-2827.

— and - 2010: Partially coherent backscatter in radar observations of precipitation. J. Atmos. Sci., 67, 1928-1946.

Kostinski, A. B., and A. Koivunen, 2000: On the condition number of Gaussian sample covariance matrices. IEEE Trans. Geosci. Remote Sens., 38, 329-332.

Lhermitte, R. M., 1960: The use of special "Pulsed Doppler Radar" in measurements of particle fall velocities. Proc. Eighth Weather Radar Conf., San Francisco, CA, Amer. Meteor. Soc., 297-302.

Sauvageot, H., 1992: Radar Meteorology. Artech House, 366 pp. 